
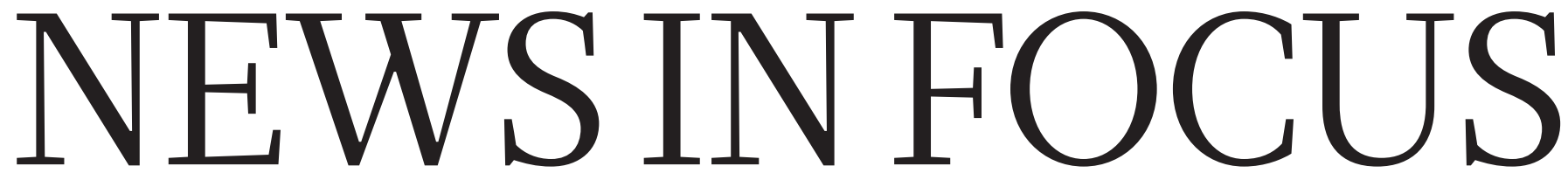

PHrsics Decades-old puzzle of how long a neutron lives

GEOPOLITICS Science embroiled in mounting China-US tensions $\mathbf{p . 4 4 3}$
BIOLOGY Geneticists make progress on mystery of how height is inherited p.444

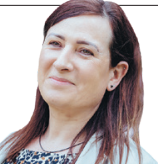

EPIDEMIOLOGY A large study in Europe tracks the health of transgender people $\mathbf{p . 4 4 6}$

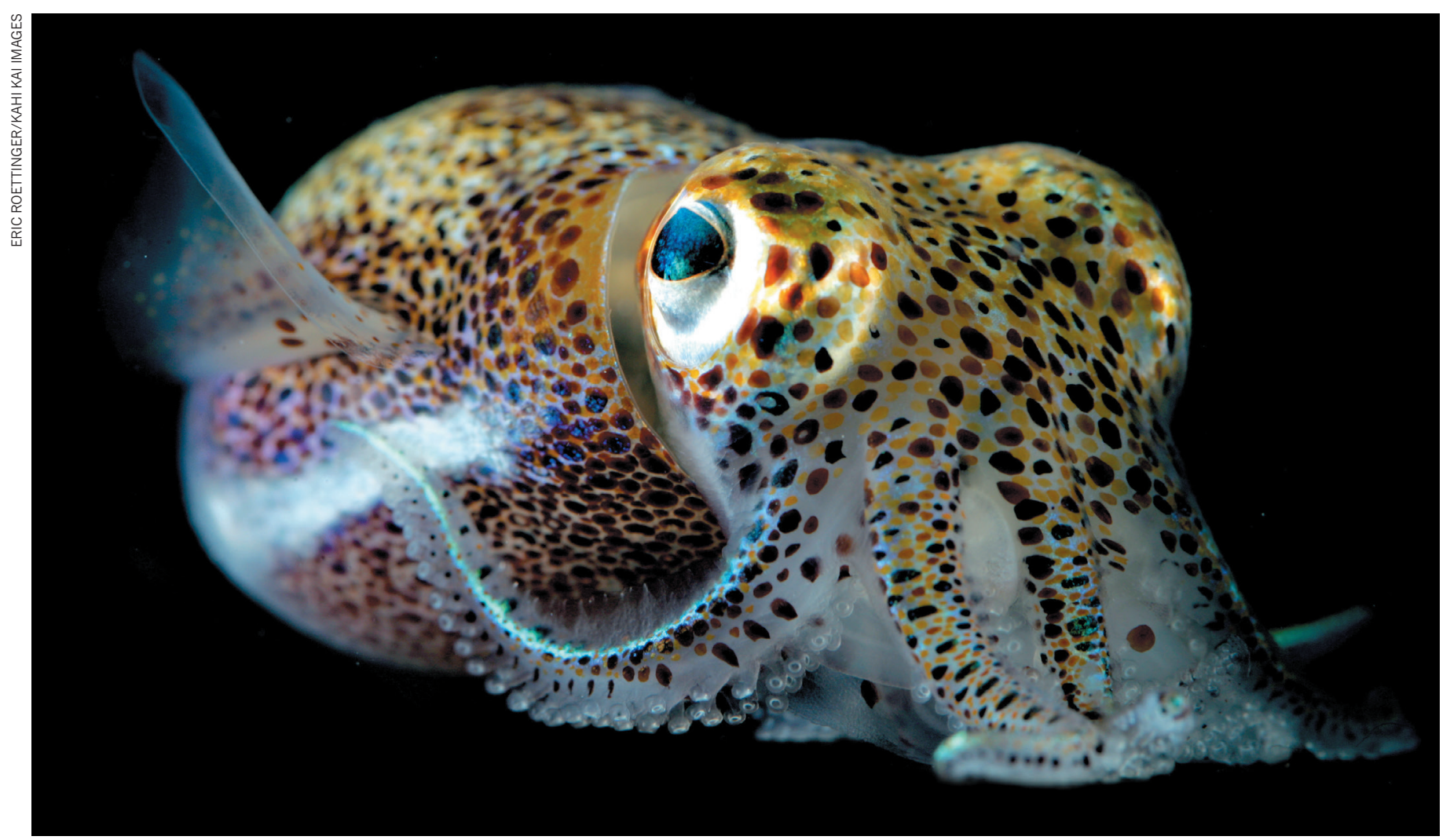

The Hawaiian bobtail squid (Euprymna scolopes) alters the camouflage patterns on its skin on the basis of what it sees.

\title{
CRISPR creates wave of exotic model organisms
}

\section{But the practical challenges of sustaining unconventional lab animals persist.}

\section{BY SARA REARDON}

Toseph Parker has wanted to know what makes rove beetles tick since he was seven years old. The entomologist has spent decades collecting and observing the insects, some of which live among ants and feed on their larvae. But without tools for studying the genetic and brain mechanisms behind the beetles' behaviour, Parker focused his $\mathrm{PhD}$ research on Drosophila fruit flies - an established model organism.
Now, more than ten years after completing his $\mathrm{PhD}$, the rise of the CRISPR gene-editing technique has put Parker's childhood dream within reach. He is using CRISPR to study symbiosis in rove beetles (Staphylinidae) in his lab at the California Institute of Technology in Pasadena. By knocking out genes in beetles that live with ants and in those that do not, Parker hopes to identify how the insects' DNA changed as their lifestyles diverged. "We're designing a model system from scratch," he says.

Biologists have embraced CRISPR's ability to quickly and cheaply modify the genomes of popular model organisms, such as mice, fruit flies and monkeys. Now, they are trying the tool on more exotic species, many of which have never before been reared in a lab or had their genomes analysed. "We finally are ready to start expanding what we call a model organism," says Tessa Montague, a molecular biologist at Columbia University in New York City. Montague works on the Hawaiian bobtail squid (Euprymna scolopes) and the dwarf cuttlefish (Sepia bandensis), species whose 
- unusual camouflage acts as an outward display of their brain activity. The cephalopods project patterns onto their skin to match what they see around them. But probing how their brains process stimuli has been difficult. Researchers would normally do this by embedding electrodes or other sensors into the skull - but squid and cuttlefish are boneless.

Last year, Montague and her team injected CRISPR components into cuttlefish and bobtail-squid embryos for the first time. Now, they are trying to genetically modify the animals' neurons to make them light up when they fire.

Other researchers are using CRISPR to study species' distinctive social behaviours. Daniel Kronauer, a biologist at the Rockefeller University in New York City, has created raider ants (Ooceraea biroi) that cannot smell pheromones. In experiments, the genetically modified ants were not able to sustain the complex hierarchy seen in a normal raider-ant colony (W. Trible et al. Cell 170, 727-735.e10; 2017). The scientists are now using CRISPR to alter genes thought to influence raider ants' behaviour.

Then there are species that threaten human or environmental health - such as the pea aphid (Acyrthosphion pisum), an insect that attacks legume crops worldwide. To edit the aphid's genome with CRISPR, a team led by Shuji Shigenobu, an evolutionary geneticist at the National Institute for Basic Biology in Okazaki, Japan, had to manipulate the insect's complex life cycle. Female aphids born in summer reproduce asexually, by cloning themselves, whereas those born in autumn lay eggs.
Shigenobu's team prompted its aphids to lay eggs by setting up an incubator that simulated the cool temperatures and short days of autumn. The scientists then injected the eggs with CRISPR components. After four years, they managed to edit a pigment gene as a proof of concept, Shigenobu announced last month during a conference at the Howard Hughes Medical Institute's Janelia Research Campus in Ashburn, Virginia.

Developing animal models requires immense amounts of time and money,

"We finally are
ready to start
expanding what
we call a model
organism."
and until recently there was little support for such work. In 2016, the US National Science Foundation launched a US\$24million programme to create model organisms - and in doing so, reveal the genetic and molecular mechanisms behind complex traits and behaviours.

The programme supports research to create tools for probing species' genomes, study organisms' life cycles and develop protocols to raise these species in the lab. This support has begun to pay off: in March, for instance, researchers at the University of Georgia in Athens said that they had used CRISPR to create the first genetically modified reptile (A. M. Rasys et al. Preprint at bioRxiv http://doi.org/c4tz; 2019).

Despite such promising early results, the push to create model organisms with CRISPR has revealed how little is known about many species' genomes, life cycles and habits. Researchers also face practical challenges, such as determining how to inject CRISPR components into embryos, and coaxing finicky, fragile species to breed in the lab.

"The reason classic model systems were chosen was they're basically pests. Nothing can stop them growing," Montague says. "But if we take on this challenge of working on new organisms because they have an amazing feature, they're often not happy to grow under [just] any conditions."

\section{INCHING FORWARDS}

This has forced scientists to weigh the effort required to study a particular trait against the potential rewards. Editing a species' genome requires a deep understanding of its behaviour and life cycle - a tall order when that organism is studied by only a few labs worldwide. "People are not choosing these model systems lightly," says David Stern, a biologist at Janelia.

Still, researchers' interest in developing atypical animal models continues to grow. Montague and her colleagues have created CHOPCHOP, a tool that allows them to design a CRISPR system for any organism. So far, scientists have sent her genetic sequences from more than 200 species, including plants, fungi, viruses and farm animals.

"I had this weekly reminder that these molecular tools do work in pretty much every organism on the planet," Montague says. "It's such an exciting time to work on any model organism - especially these new and weird creatures." -

\section{Physicists close in on neutron puzzle}

\section{Researchers are narrowing down their measurements of how long the subatomic particle survives on its own.}

\section{BY ALEXANDRA WITZE IN DENVER, COLORADO}

$\mathrm{P}$ hysicists are drawing nearer to answering a long-standing mystery of the Universe: how long a neutron lives.

Neutrons are electrically neutral particles that usually combine with protons to make up atomic nuclei. Some neutrons are not bound up in atoms; these free-floating neutrons decay radioactively into other particles in minutes.

But physicists can't agree on precisely how long it takes a neutron to die. Using one laboratory approach, they measure the average neutron lifetime as 14 minutes 39 seconds. Using a different approach, they get 8 seconds longer.
"We don't know why they're different," says Shannon Hoogerheide, a physicist at the National Institute of Standards and Technology (NIST) in Gaithersburg, Maryland. "We really need to understand and eliminate this discrepancy." She and other scientists debated new ways to solve the problem this month at a meeting of the American Physical Society in Denver, Colorado.

Pinpointing the lifetime of a neutron is important for understanding how much hydrogen, helium and other light elements formed in the first few minutes after the Universe was born 13.8 billion years ago. Scientists also think that pinning down the neutron's lifetime would help to constrain measurements of other subatomic particles.

One way of clocking the neutron's lifespan is to put some of the particles in a bottle and count how many are left after a period of time. This 'bottle' method has been tried at several laboratories, including the Los Alamos National Laboratory in New Mexico ${ }^{1}$ and the Institut Laue-Langevin in Grenoble, France. On average, they come up with a neutron lifetime of 14 minutes 39 seconds.

The other way is to feed neutrons into a detector that counts the protons created as the neutrons decay. This 'beam' method has been used at NIST and at the Japan Proton Accelerator Research Complex in Tokai. The Japanese work has just begun, but the NIST team reported in 2013 that its neutrons live eight seconds longer, on average, than those in the bottle method ${ }^{2}$.

That's a big problem, because the beam and bottle measurements don't overlap, even when their margins of error are taken into account. So physicists have been looking for ways to explain why neutrons might be disappearing from bottles faster than from beams.

One possibility is that one of the two methods is doing something wrong. In that case, researchers might want to combine beam 